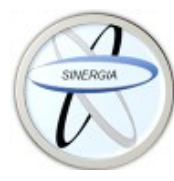

Revista Médica Sinergia

Vol. 6, Núm. 5, mayo 2021, e673

https://doi.org/10.31434/rms.v6i5.673

revistamedicasinergia@gmail.com

\title{
Trombosis de los senos venosos
}

\section{Venous sinus thrombosis}

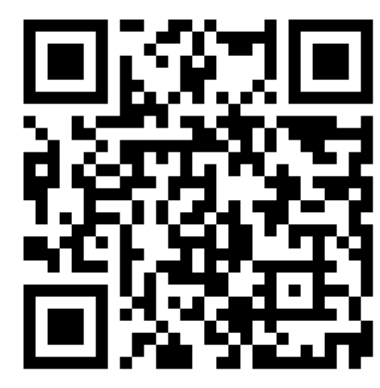

Recibido 03/03/2021
Corregido $04 / 04 / 2021$

${ }^{1}$ Dra. Priscilla Montoya Alan Investigadora independiente, San José, Costa Rica

(D) https://orcid.org/0000-0002-0216-8961

${ }^{2}$ Dra. Katherine Murillo Alvarado Investigadora independiente, Alajuela, Costa Rica

(iD) https://orcid.org/0000-0002-7156-4554

${ }^{3}$ Dra. Mariel Morales Fallas Investigadora independiente, San José, Costa Rica

(iD) https://orcid.org/0000-0001-7151-1359

\section{RESUMEN}

Los senos venosos son los encargados de drenar la sangre cerebral hacia la vena yugular interna.

La trombosis de estos senos es poco común y ocurre mayormente en mujeres de edad fértil o en estado de embarazo. La sintomatología que se produce en este cuadro clínico se debe al aumento de la presión intracraneal por lo cual lo más común son cefalea, papiledema y déficit motor. Entre las causas más comunes que producen esta patología están los estados protrombóticos y los cuadros infecciosos. El diagnostico se realiza mediante imagenología entre las cuales están la resonancia magnética y la tomografía axial computarizada. El tratamiento dependerá de la causa, si es un trastorno de coagulación, se darán anticoagulantes y si es por un cuadro infeccioso se cubrirá con antibióticos.

PALABRAS CLAVE: cefalea; presión intracraneal; convulsiones; embarazo; sangre.

\section{ABSTRACT}

The venous sinuses are responsible for draining the cerebral blood into the internal jugular vein. Thrombosis of these breasts is rare and occurs mostly in women of childbearing age or in pregnancy. The symptoms that occur in this clinical picture are due to increased intracranial pressure, which is why the most common are headache, papilledema and motor deficit. Among the most common causes that produce this pathology are prothrombotic states and infectious pictures. The diagnosis is made by imaging, including magnetic resonance imaging and computerized axial tomography. Treatment will depend on the cause, if it is a clotting disorder, 
anticoagulants will be given and if it is due to an infectious condition, it will be covered with antibiotics.

KEYWORDS: headaches; intracranial pressure; seizures; pregnancy; blood.

${ }^{1}$ Médica general, graduada de la Universidad de Ciencias Médicas (UCIMED). Cód. MED13645. Correo: priella86@gmail.com

${ }^{\mathbf{2}}$ Médica general, graduada de la Universidad de Ciencias Médicas (UCIMED). Cód. MED12617. Correo: Kat.murilloalvarado@gmail.com

${ }^{3}$ Médica general, graduada de la Universidad de Ciencias Médicas (UCIMED). Cód. MED13964. Correo: mari mo 1@hotmail.com

\section{INTRODUCCIÓN}

Los senos venosos dúrales son canales trabeculares grandes revestidos de endotelio que colecta la sangre cerebral a nivel superficial, profunda y fosa posterior, debido a su recorrido intracraneal $y$ finalmente hacia su drenaje. Excretan el líquido cefalorraquídeo (LCR) por medio de las granulaciones aracnoideas $(1,2)$.

La trombosis de senos venosos es un tipo de accidente vascular cerebral (AVC) infrecuente, la cual afecta los grandes senos venosos y las venas cerebrales, fue descrita por el médico francés $M$. Ribes, quién en 1825 describió a un paciente con cefalea intensa, delirio y convulsiones.

ocasionada mayormente en mujeres especialmente en edades fértiles y en el periodo de embarazo y postparto. Con una incidencia anual entre 0.22 a 1,57 por 100 000 habitantes. Ocasionan el 0.5 a $1 \%$ de los AVC (2-5).

Los factores de riesgo sean genéticos por coagulopatías 0 adquiridos estados protrombóticos como el uso de anticonceptivos orales (ACO), embarazo, puerperio, infecciosas. Por lo anterior, la etiología sea aséptica o séptica (secundaria a infecciones e infrecuentes) (3-7).

La sintomatología explicada por su patogenia de aumento de la presión intracraneal y estructuras anatómicas, aunque es inespecífica se desataca la cefalea en un $80 \%$ de los casos y papiledema en un $50 \%$, convulsiones, déficits motores y sensitivos, afección de los pares craneales III, IV y IV principalmente a la afección del seno cavernoso secundario a otitis o sinusitis $(2,4,6,8-9)$.

El diagnóstico es realizado por resonancia magnética (RM) y tomografía axial computarizada (TAC), que su visualización dependerá de su tiempo de evolución y su tratamiento debe ser instaurado inmediatamente el tratamiento que ha mejorado el pronóstico en un gran porcentaje de los casos, se inicia con encontrar la causa subyacente (si la posee), control de la clínica y prevención de complicaciones como tratamiento antitrombótico y anticoagulante 0 administración antibiótica cuando lo es requerido, donde se ha reducido de un $100 \%$ a $30 \%$ en la mortalidad $(3,10-12)$.

El objetivo de este artículo es realizar una revisión del tema con bibliografía actualizada enfocado en los factores de riesgo, causas y clínica, así como las herramientas diagnósticas y tratamiento oportuno que influyen directamente sobre la morbi mortalidad del paciente.

\section{MÉTODO}

En esta revisión se investigó y repasó el tema de trombosis de senos venosos con sus generalidades y se escogieron bibliógrafías entre los años 2017 hasta el 2021, las cuales abarcan desde libros de texto, revistas digitales como Elsevier, así como páginas web especializadas en medicina como UpToDate.

Se utilizó búsqueda entre los temas "senos venosos", "trombosis de senos venosos", "trombosis y embarazo", "infecciones y 
senos venosos"; donde toda esta información que se obtuvo, se hizo un detallado análisis y se seleccionó lo más relevante y de mayor importancia científica para brindar una revisión de calidad.

\section{GENERALIDADES DEL SISTEMA VENTRICULAR}

El recorrido del drenaje se encuentra entre el seno sagital superior, seno recto, seno transverso y seno occipital drenan hacia la confluencia de los senos. El seno sagital inferior drena hacia el seno recto y los senos petrosos superior e inferior, drenan hacia el seno sigmoideo, e infrecuentemente el seno potroso superior drena en el seno transverso (1).

Contienen relaciones anatómicas importantes como lo es en el interior del seno cavernoso recorre la porción cavernosa de la arteria carótida interna junto con el sexto par craneal (nervio abducens). Y en la pared lateral se encuentra el nervio oculomotor (III), nervio troclear (IV), divisiones de nervio trigémino $(\mathrm{VI})$ : nervio oftálmico $(\mathrm{V} 1)$ y nervio mandibular (V2) (1).

Dentro de la estructuración venosa se encuentran las venas cerebrales, las cuales no contienen válvulas, por lo que un flujo bidireccional es posible y se dividen en tres grupos (1):

\section{1) Sistema venoso superficial}

\section{a) Vena cortical superior}

- Drenan en el Seno sagital inferior

- La más conocida es la Vena de Trolard, sin embargo, hay un 8 a 12 vasos pequeños que también conforman parte.

\section{b) Vena cortical media}

- Drenan al Seno cavernoso.

- Reciben de la parte inferior del lóbulo frontal, giro temporal superior y opérculo parietal.
- La vena de Labbé es la representativa

\section{c) Vena cortical inferior}

- La mayor vena es la vena cerebral media profunda.

- Reciben de lóbulo frontal inferior y lóbulo temporal (ínsula, ganglios basales y giro parahipocampal).

- La vena basal de Rosenthal (VBR) pertenece a este grupo.

\section{2) Sistema venoso profundo}

Cómo se describe en su nombre, drenan los hemisferios cerebrales profundos, incluyendo los ganglios de la base, cuerpo calloso, tálamo y sistema límbico.

Es drenado por la vena cerebral interna que junto a la VBR forman la gran vena galena donde termina en el seno recto, y así continúa con el recorrido.

3) Venas del tallo cerebral y fosa posterior. Se dividen en tres grupos: superior, anterior y posterior.

Figura 1. Senos dúrales venosos

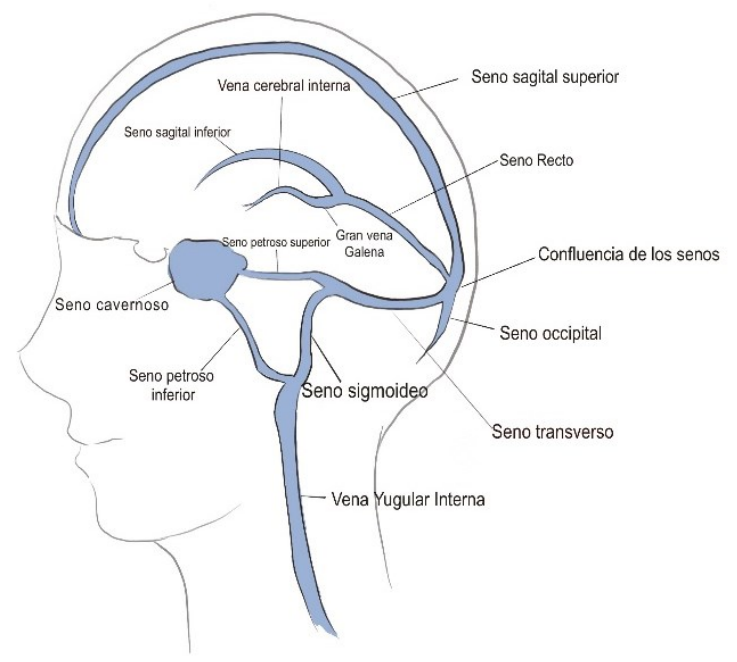

Fuente. Figura realizada por Dra. Priscilla Montoya Alán 


\section{EPIDEMIOLOGÍA}

Es una etiología poco común dentro de las variantes de los AVC.

Más frecuente en mujeres que hombres (3:1), (3) y predomina en edades 20 a 35 años, especialmente durante el embarazo y post parto (6).

La variante séptica es más infrecuente que la aséptica (13). Siendo el seno cavernoso más frecuente en la variante séptica y el seno sagital superior el más afectado en un $70 \%$ de los casos en la aséptica $(6,9)$.

Sin tratamiento puede ser mortal, sin embargo; posee un buen pronóstico

\section{PATOGENIA}

Las causas predisponentes son diversas y desconocidos en su totalidad, sin embargo, comparten uno de los dos mecanismos fundamentales el cual es la lesión de la pared endotelial del seno venoso o la descompensación congénita o adquirida de la capacidad trombótica y trombótica de la sangre $(6,7)$.

Los mecanismos fisiopatológicos explican la clínica sí hay obturación de los senos venosos hay acúmulo de sangre y LCR dañando el parénquima cerebral y terminando en hipertensión intracraneal $(3,7,14)$.

Consecuentemente, la oclusión de las venas corticales, aumentando la presión venocapilar que termina en ruptura de barrera hematoencefálica, según describe Ferro, J. $M$ et all, "La ruptura causa edema vasogénico, con derrame sanguíneo al espacio intersticial.

Así como el incremento de presión puede llevar al incremento de la presión intravascular ocasionando disminución de la perfusión cerebral y falla metabólica. Esto permite, la entrada de líquido intracelular por la falla de la bomba $\mathrm{Na}+/ \mathrm{K}+$ resultado edema citotóxico" (7).

\section{FACTORES DE RIESGO Y ETIOLOGÍA}

En la tabla 1, se especifican los factores de riesgo adquiridos $y$ genéticos que predisponen al paciente y no deben ser descartados al enfrentarse a esta patología. En un $85 \%$ de los casos en pacientes adultos, se debe a una condición protrombótica y en una minoría $<10 \%$ no se tiene causa identificable.

La etiología se dividirá en trombosis asépticas y sépticas (12). Ya que, por su proceso fisiopatológico y su tratamiento concomitante, identificar la causa adyacente es primordial, sea asépticas debido a el estado protrombótico adquirido o genética o séptico.

En cuanto al cuadro séptico lo podemos dividir en infecciones localizadas 0 generalizadas:

1) Locales $(6,9)$

- Trauma séptico directo.

- Intracraneal (absceso, empiema, meningitis).

- Localizada (otitis, tonsilitis, sinusitis, estomatitis, cutánea), siendo los agentes causales en un $70 \%$ de los casos Staphylococcus aureus, seguido de Streptococos, gram negativo, anaerobios y hongos.

2) General $(6,9)$

- Bacterianas: septicemia, endocarditis, tuberculosis

- Víricas: sarampión, hepatitis, encefalitis, herpes, citomegalovirus. Virus inmunodeficiencia humana (HIV)

- Parasitarias: malaria, triquinosis

- Fúngicas: aspergilosis

De manera novedosa, reporta Khacha, A et all., se han reportado casos de trombosis de 
los senos venosos cerebrales relacionados con el nuevo virus SARS CoV-2 que ocasiona el COVID 19, se anota con mayor afección el seno sagital superior (15).

\section{CLÍNICA}

Depende factores como la edad y el sexo, el sitio, el número de senos y venas ocluidas, si se presenta lesión en parénquima cerebral o no. Además de si el curso es agudo o crónico.

Puede ser agrupada en tres grandes síndromes (6):

- Hipertensión intracraneal aislada. Cefalea, papiledema, vómitos.

- Síndrome focal. Déficit focal, convulsiones o ambos.

- Encefalopatía. Estupor, coma, cambios en el estado mental.
Presentaciones menos comunes se encuentran $(3,6)$ :

- Síndrome del seno cavernoso

- Hemorragia subaracnoidea

- Parálisis múltiples de nervios craneales.

Los niños presentan signos de daño cerebral difuso, coma y convulsiones (especialmente los neonatos (6). Los niños mayores su clínica ya es similar a los adultos con la cefalea y hemiparesias.

Existe un $10 \%$ compuesto por hombres, ancianos, pacientes con neoplasia y trombosis aislada de la vena cortica que se cursa con ausencia total de cefalea (3).

En la tabla 2 se describen los signos de manera individual.

TABLA 1. Factores de riesgo adquiridos y genéticos.

\begin{tabular}{|c|c|}
\hline Riesgos adquiridos & Riesgos genéticos \\
\hline $\begin{array}{l}\text { 1. Anticonceptivos orales que contienen } \\
\text { estrógeno } \\
\text { 2. Embarazo } \\
\text { 3. Puerperio } \\
\text { 4. Neoplasias } \\
\text { 5. Infecciones } \\
\text { 6. Vasculitis } \\
\text { 7. Deshidratación } \\
\text { 8. Alteraciones del sistema nervioso central } \\
\text { 9. Trauma craneoencefálico (menos } \\
\text { frecuente) }\end{array}$ & $\begin{array}{l}\text { 1. Trombofilias hereditarias incluidas: } \\
\text { deficiencias de antitrombina } \\
\text { - Proteína C } \\
\text { - Proteína S } \\
\text { - Mutación del factor V de Leyden } \\
\text { - Mutación del gen } 20210 \text { de } \\
\text { - Hiperombina }\end{array}$ \\
\hline \multicolumn{2}{|c|}{$\begin{array}{l}\text { Fuente. Otárola Valderrama, S., Salazar Rosa, V., \& Trujillo Santos, J. (2020). Compendio de enfermedad } \\
\text { tromboembólica venosa (1. }{ }^{a} \text { ed., pp. 129-131). Elsevier Inc. Elsevier Inc. } \\
\text { Polo Verbel L, Torres Zambrano M, Fernández Yépez L, Escorcia Barceló O, López Roa LM. Trombosis de senos } \\
\text { venosos cerebrales, una manifestaciónón inusual de déficit de proteína S en paciente joven. Acta Neurol Colomb } \\
\text { [Internet]. } 2016 \text { [citado } 2 \text { enero 2020];32(4):305-309. Disponible en: http://dx.doi.org/10.22379/24224022112. } \\
\text { Ferro J, Canhão P. Cerebral venous thrombosis: Etiology, clinical features, and diagnosis [Internet]. UpToDate. } \\
2020 \text { [citado } 3 \text { enero 2021]. Disponible en: } \text { https://www-uptodate-com.binasss.idm.oclc.org/contents/cerebral- } \\
\text { venous-thrombosis-etiology-clinical-features-and- } \\
\text { diagnosis?search=sinus\%20thrombosis\&source=search result\&selectedTitle=1 150\&usage type=default\&display } \\
\text { rank=1\#H3 }\end{array}$} \\
\hline
\end{tabular}


Tabla 2. Signos de Trombosis de los senos venosos

\begin{tabular}{|c|c|}
\hline Signo & Descripción \\
\hline Cefalea & $\begin{array}{l}\text { - La cefalea siendo más del } 80 \% \text { de los casos el síntoma inicial, se } \\
\text { presenta como una nueva cefalea o parte de un síndrome de } \\
\text { hipertensión intracraneal }(5,6) \text {. } \\
\text { - No hay relación del sitio de la cefalea con el seno ocluido }(6) \text {. } \\
\text { - Es gradual e insidiosa, tendencia cronificar }(3,6) \text {. }\end{array}$ \\
\hline $\begin{array}{l}\text { Síndrome de } \\
\text { hipertensión } \\
\text { intracraneal } \\
\text { aislada (SHIC) }\end{array}$ & $\begin{array}{l}\text { - Cefalea + papiledema o problemas visuales } \\
\text { - Ocurre en gran cantidad de los pacientes. }\end{array}$ \\
\hline Convulsiones & $\begin{array}{l}\text { - } \text { Pueden ser focales o generalizadas } \\
\text { - Un } 40 \% \text { de los casos, mayormente fase aguda (3) }\end{array}$ \\
\hline Encefalopatía & $\begin{array}{l}\text { - Alteración del estado de consciencia y disfunción cognitiva como } \\
\text { delirium, apatía, déficit multifocales (6). }\end{array}$ \\
\hline Síndrome focal & $\begin{array}{l}\text { - Debilidad con monoparesis o hemiparesis } \\
\text { - Bilateral en algunas ocasiones } \\
\text { - Es el déficit focal más frecuente }\end{array}$ \\
\hline $\begin{array}{l}\text { Trombosis } \\
\text { aisladas }\end{array}$ & $\begin{array}{l}\text { - Seno cavernoso } \\
\text { - Si es séptico se acompaña de fiebre } \\
\text { - Parálisis del III, IV y VI par craneal. } \\
\text { - Proptosis } \\
\text { - Disminución o ausencia del reflejo corneal (8) }\end{array}$ \\
\hline & $\begin{array}{l}\text { - Seno sagital superior } \\
\text { - Déficit motor, bilaterales } \\
\text { - Convulsiones son frecuentes }\end{array}$ \\
\hline & $\begin{array}{l}\text { - Seno transverso } \\
\text { - Cefalea o SHIC aislada } \\
\text { - Menos frecuente: déficits focales o convulsiones }\end{array}$ \\
\hline & $\begin{array}{l}\text { - Vena yugular } \\
\text { - Tinnitus pulsátil }\end{array}$ \\
\hline \multicolumn{2}{|c|}{$\begin{array}{l}\text { Fuente. Realizada por Priscilla Montoya Alán en base a: } \\
\text { 'Otárola Valderrama, S., Salazar Rosa, V., \& Trujillo Santos, J. (2020). Compendio de enfermedad tromboembólica venosa (1. a ed., } \\
\text { pp. 129-131). Elsevier Inc. Elsevier Inc. } \\
\text { Ferro J, Canhão P. Cerebral venous thrombosis: Etiology, clinical features, and diagnosis [Internet]. UpToDate. } 2020 \text { [citado } 3 \text { enero } \\
\text { 2021]. Disponible en: https://www-uptodate- com.binasss.idm.oclc.org/contents/cerebral-venous-thrombosis-etiology-clinical- } \\
\text { features-and- diagnosis? search=sinus\%20thrombosis\&source=search_result\&selectedTitle=1 150\&usage_type=defau } \\
\text { lt\&display_rank=1\#H3 } \\
\text { 2Choi K, Yang C. A case report of cavernous sinus thrombosis after trauma. International Journal of Pediatric Otorhinolaryngology } \\
\text { [Internet]. } 2017 \text { [citado } 2 \text { enero 2021];(95):101-103. Disponible en: http://dx.doi.org/10.1016/i.jiporl.2017.02.007 }\end{array}$} \\
\hline
\end{tabular}




\section{DIAGNÓSTICO}

Al ser una patología con una variedad de síntomas, usualmente se tarda en el diagnóstico.

Se debe considerar en pacientes menores de 50 años con cefaleas agudas, subagudas o crónicas y que tengan características atípicas, incluyendo déficit neurológico focal, convulsiones, signos de hipertensión intracraneal, puede haber papiledema en el examen de fondo de ojo, pero no es sensible ni específico (19).

En los exámenes de laboratorio puede estar elevado el leucograma (si se piensa en origen infeccioso como la causa) y también se debe evaluar el dimero D, sin embargo, una prueba de dímero d no puede descartar la afección, ya que en un estudio se documentó una tasa de falsos negativos del $24 \%$ y una tasa de falsos positivos del $9 \%$ en una cohorte de 239 pacientes; por lo que no puede un dímero d normal no se debe utilizar para para descartar una trombosis (19).

La realización de una punción lumbar para analizar el LCR debe indicarse con cuidado si hay hipertensión endocraneal y solo está claramente justificada si hay indicios clínicos de una meningitis asociada y es preciso realizar un cultivo del LCR.

EI LCR revela hallazgos inespecíficos como una leve pleocitosis linfocitaria y ligero aumento de proteínas y hematíes (30-50\% de los casos) 20.

La imagenología es el método que se utiliza para realizar el diagnóstico y se puede utilizar las siguientes:

La tomografía axial computarizada (TAC) puede ser normal hasta en el $30 \%$ de los casos de infartos venosos corticales y se puede observar (20):

- Signos del infarto cortical que asocia áreas de hemorragia, con edema y posible HSA de la convexidad.
- Edema desproporcionado al área de infarto. El edema generalizado con ventrículos pequeños se observa en la trombosis del SLS con HIC

- Bilaterales y no siguen ningún territorio arterial.

- Hipodensidad bilateral de los ganglios basales, lo cual indica una trombosis de la vena de Galeno.

Las imágenes directas de la trombosis venosa son muy raras en la tomografía y se pueden observar dos signos (20):

- Signo de la cuerda: es la imagen que produce la vena trombosada espontáneamente hiperdensa.

- Signo del delta: es la imagen que se produce, tras la inyección de contraste, en la porción posterior del SLS cortado de manera transversal, como de una tienda de campaña con su interior hipodenso ocupado por el trombo.

La resonancia magnética (RM) es más sensible para detectar los infartos venosos corticales y depende del tiempo de evolución, así se visualizará el trombo (3):

- En menos de 5 días: trombosis isointensas en T1 e hipointensos en T2.

- Mayor de 5 días: trombos isointensos en T1 y T2.

- Mayor de 1 mes: señal variable.

De manera directa se observará un defecto de llenado y agrandamiento del seno afectado $(8,12)$.

Indirectamente dependiendo la causa ya sea infecciosa se puede observar edema orbital, exoftalmos, entre otros (12). 
En casos de trombosis séptica, Bathia, $\mathrm{H}$ et all, llegaron a la conclusión de los siguientes parámetros (15):

- Seno cavernoso mayor o igual de 10 $\mathrm{mm}$ de diámetro.

- Vena oftálmica superior mayor o igual de $2.9 \mathrm{~mm}$ de diámetro

- Arteria carótida interna comprimida en menor o igual de $4.2 \mathrm{~mm}$ de diámetro

La RM se puede combinar con angio-RM, que permite observar de forma clara los grandes senos y las principales venas corticales. En los pacientes con una oclusión total del seno visible en la RM es posible que no sea necesaria la angio-RM, pero las oclusiones parciales pueden ser sintomáticas (HIC) y, aunque la RM sea normal, la angio-RM es obligatoria (20).

\section{TRATAMIENTO}

El manejo se debe enfocar en primera instancia en abordar primero las complicaciones potencialmente mortales, que incluyen compromiso respiratorio, convulsiones o aumento de la PIC. Estos procedimientos van seguidos de un tratamiento específico según la causa de la trombosis, cuidados de apoyo y manejo de las complicaciones subyacentes

Cuando la causa se debe a una trombosis el tratamiento se basa en el uso de los anticoagulantes como la heparina para prevenir la progresión del trombo (16), la eficacia de estos está avalada por estudios controlados y no se ha demostrado que la anticoagulación empeore los infartos hemorrágicos 19.

Para los pacientes que no tienen mejoría con la heparina se han publicado tratamientos endovasculares complejos valorados para cada caso y que incluyen la fibrinólisis local, la aspiración del trombo y la remodelación/angioplastia del seno con colocación de stent; también la craniectomía descompresiva amplia para evitar la herniación y el coma se reserva para los casos que cursan con HIC refractaria a las otras medidas (20).

No está bien establecido durante cuánto tiempo hay que mantener la anticoagulación, primero con HBPM o heparina fraccionada y luego con cumarínicos. En adultos se recomienda de 3 a 12 meses, y en niños, de 3 a 6 meses, con un INR de 2-3 y solo se justifica continuar con la anticoagulación si hay trombosis recidivante o alguna causa que predispone a la trombosis (trastornos de la coagulación, enfermedad de Behcer u otra patología).

Los pacientes que sobreviven al ictus venoso agudo pueden quedar con diversas secuelas, como defectos cognitivos 0 focales, o epilepsia. Usualmente las secuelas de los infartos venosos son menores que las de los infartos arteriales, la epilepsia crónica es rara, por lo que no está justificado un tratamiento antiepiléptico definitivo. (20)

El tratamiento cuando la causa es por algún agente infeccioso se debe cubrir con antibióticos intravenosos, realizar drenajes quirúrgicos o lavados (17).

Cuando se trata de infecciones orbitarias (celulitis 0 abscesos subperiósticos $u$ orbitarios) deben recibir tratamiento inmediato con antibióticos intravenosos y vigilar muy de cerca que no se presenten signos de afectación visual. En todos los casos se deben extraer muestras para hemocultivo, y también de los senos paranasales en los pacientes con sinusitis.

El tratamiento inicial debe consistir en antibióticos de amplio espectro como vancomicina, metronidazol y una cefalosporina de tercera o cuarta generación, para tener una cobertura frente a $S$. aureus, incluido SARM, estreptococos, incluido $S$. pneumoniae, $H$. influenzae y otros bacilos gramnegativos y anaerobios (17). 
El tratamiento antibiótico debe personalizarse en función de los resultados de los cultivos ( $p$. ej., de sangre, de los senos, del drenaje del absceso); además cuando se documenta algún absceso ya sea subperióstico, cerebral, dentario, retro ocular, por sinusitis; se debe proceder con rapidez al drenaje quirúrgico del absceso aparte del tratamiento con antibióticos de amplio espectro (18).

\section{PRONÓSTICO}

La mortalidad oscila entre el 5 y el $30 \%$, y depende mucho de las características de la serie. La mortalidad más alta se da entre los pacientes que necesitan ingreso en la unidad de cuidados intensivos con alteraciones de la consciencia, infartos hemorrágicos profundos o expansivos con desplazamientos y hernias (20).

\section{CONCLUSIONES}

La trombosis de senos venosos es un tipo de accidente cerebral vascular poco común y con una sintomatología diversa por lo cual se debe tener en cuenta siempre que se valoren mujeres jóvenes en estado fértil, embarazo o personas con enfermedades que causen trastornos de coagulación.

Esta patología puede ser desencadenada por varias causas, por lo cual es de suma importancia distinguir si es por causas asépticas como el uso de anticonceptivos orales, embarazo, puerperio, cáncer, trastornos congénitos de la coagulación o las causeas sépticas como los procesos infecciosos como la meningitis, sinusitis, otitis, etc. ya que dependiendo de la causa así se enfocará el tratamiento a seguir; ya sea utilizando anticoagulantes o antibioticoterapia.

Actualmente gracias a los avances en las tecnologías radiológicas, el diagnostico se puede hacer de una manera más expedita por lo cual evitamos hacer un diagnóstico tardío; con lo cual disminuimos la mortalidad y evitamos secuelas neurológicas graves en los pacientes.

\section{Los autores declaran no tener conflicto de interés.}

\section{REFERENCIAS}

1. Caplan, L. R., Biller, J., Leary, M., Lo, E., Thomas, A., Yenari, M., \& Zhang, J. (2017). Primer on Cerebrovascular Diseases (2. ${ }^{a}$ ed., pp. 32-36). Elsevier Inc. Elsevier Inc.

2. Goldman, L., \& Schafer, A. (2020). Goldman-Cecil Medicine (26. ${ }^{a}$ ed., pp. 2458-63). Elsevier Inc. Elsevier Inc.

3. Otárola Valderrama, S., Salazar Rosa, V., \& Trujillo Santos, J. (2020). Compendio de enfermedad tromboembólica venosa (1. ${ }^{\mathrm{a}}$ ed., pp. 129-131). Elsevier Inc. Elsevier Inc.

4. Polo Verbel L, Torres Zambrano M, Fernández Yépez L, Escorcia Barceló O, López Roa LM. Trombosis de senos venosos cerebrales, una manifestación inusual de déficit de proteína $S$ en paciente joven. Acta Neurol Colomb [Internet]. 2016 [citado 2 enero 2020];32(4):305-309. Disponible en: http://dx.doi.org/10.22379/24224022112

5. Rozman C, Cardellach F. Medicina Interna. 19. ${ }^{a}$ ed. Elsevier Inc.; 2020.

6. Ferro J, Canhão P. Cerebral venous thrombosis: Etiology, clinical features, and diagnosis [Internet]. UpToDate. 2020 [citado 3 enero 2021]. Disponible en: https://wwwuptodate.com.binasss.idm.oclc.org/contents/cere bralvenous-thrombosis-etiology-clinical-featuresand-

diagnosis?search=sinus $\% 20$ thrombosis $\&$ source $=$ search_result\&selectedTitle=1 150\&usage_type =default \&display_rank=1\#H3

7. Southwick FS. Septic dural sinus thrombosis [Internet]. UpToDate. 2020 [citado 3 enero 2021]. Disponible en: https://www-uptodatecom.binasss.idm.oclc.org/contents/septic-duralsinus-thrombosis?

search=sinus \%20thrombosis\&source=search_re sult\&selectedTitle=3 150\&usage_type=default \&display_rank=3\#H1319409772

8. Choi K, Yang C. A case report of cavernous sinus thrombosis after trauma. International Journal of Pediatric Otorhinolaryngology [Internet]. 2017 [citado 2 enero 2021];(95):101-103. Disponible en: http://dx.doi.org/10.1016/j.ijporl.2017.02.007

9. López F, Santamarta E, Martínez P, Sáiz-Ayala A, Llorente J. Cavernous sinus thrombosis during pregnancy. Auris Nasus Larynx [Internet]. 2017 
[citado 2 enero 2021];(44):232-236. Disponible en: http://dx.doi.org/10.1016/j.anl.2016.04.006

10. Ferro J, Canhão P. Cerebral venous thrombosis: Treatment and prognosis. UpToDate. 2020 [citado 3 enero 2021]. Disponible en: https://wwwuptodate-com.binasss.idm.oclc.org/contents/ cerebral-venous-thrombosis-treatment-andprognosis? search $=$ sinus $\% 20$ thrombosis \&topicRef $=1103 \&$ so urce=see_link\#H265486957

11. Geng B, Wu X, Malhotra A. Septic cavernous sinus thrombosis-Case series and review of the literature. Clinical Neurology and Neurosurgery [Internet]. 2020 [citado 5 enero 2021]; (197):1-6. Disponible en: https://doi.org/10.1016/j.clineuro.2020.106092

12. Battisitn U, Hallak $Y$, Hallak F, Hallak $O$, Alkhani R. Subacute Cavernous Sinus Thrombosis following a Dental Procedure: Case T Report and Review of the Literature. Clinical Neurology and Neurosurgery [Internet]. 2020 [citado 5 enero 2020];(197):1-10. Disponible en: https://doi.org/10.1016/j.clineuro.2020.106135

13. Zarranz J. Neurología. 6. ${ }^{a}$ ed. Elsevier; 2018.

14. Khacha A, Bouchal S, Ettabyaoui A, Haloua M, Alaoui Lamrani $\mathrm{Y}$, Boubbou $\mathrm{M}$, Belahsen $\mathrm{F}$, Maâroufi M, Alami B. Cavernous sinus thrombosis in a COVID-19 patient: A case report. Radiology case reports [Internet]. 2021 [citado 3 enero 2021];(16):480-482. Disponible en: https://doi.org/10.1016/j.radcr.2020.12.013

15. Bhatia $H$, Kaur $R$, Bedi $R$. MR imaging of cavernous sinus thrombosis. European Journal of Radiology Open [Internet]. 2020 [citado 4 enero 2020];(7). Disponible en: https://doi.org/10.1016/j.ejro.2020.100226

16. Kumar A, Saroch A, Sharma N. Danger triangle of face and septic cavernous sinus trombosis. The Journal of Emergency Medicine [Internet]. 2017 [citado 4 enero 2020]; (53):137-138. Disponible en:

http://dx.doi.org/10.1016/j.jemermed.2017.03.016

17. Hupp, J., \& Ferneini, E. (2017). Infecciones orofaciales, de cabeza y cuello (1. ${ }^{a}$ ed.). España: Elsevier. España: Elsevier.

18. Bennett, J., Dolin, R., \& Blaser, M. J. (2020). Enfermedades infecciosas. Principios y práctica (9. ${ }^{a}$ ed.). España: Elsevier. España: Elsevier

19. Gurgel , R. K., \& Harnsberger, R. (2018). Imaging in Otolaryngology (1. ${ }^{a}$ ed.). España: Elsevier. España: Elsevier.

20. Zarranz, J. J. (2018). Neurología (6. ${ }^{a}$ ed.). España: Elsevier. España: Elsevier. 